\title{
A Study on Optimal D-InSAR Filtering Technique According to Landform Relief
}

\author{
Youm Minkyo, Yun Hongsic *, Kim Kwangbae, Kim Hanbual and Lee Woneung \\ Interdisplinary Program in Crisis, Disaster and Risk Management, Sungkyunkwan University, Suwon 2066, \\ Korea; tomsmith850918@gmail.com (Y.M.); kbkim929@gmail.com (K.K.); onestar1015@gmail.com (K.H.); \\ skinid777@skku.edu (L.W.) \\ * Correspondence: yoonhs@gmail.com; Tel.: +82-031-290-7536
}

\begin{abstract}
The InSAR technique measures the displacement of an indicator using SAR image interference. The technique of interfering two SAR images among InSAR techniques is called Differential InSAR (D-InSAR). In the process of D-InSAR, the filtering technique is used in the Unwrapped Mask, usually using the GoldStein method. However, since the Goldstein method removes the noise on the path, it is difficult to derive the displacement value in the agricultural area where the relative coherence is low. In Korea, more than $50 \%$ of the whole country consists of mountainous regions and agricultural regions, so it is difficult to use the Goldstein technique polysynthetically. In this study, we set the test-bed for the urban area and the agricultural area based on Coherence, and introduce Goldstein and Boxcar Filter, one of the Goldstein and LSM techniques. Through this process, we want to draw the conclusion which is displacement values in the agricultural area.
\end{abstract}

Keywords: D-InSAR; Coherence; Goldstein; Boxcar-filter

\section{Introduction}

The largest earthquake occurred in Gyeong-ju since the observation on the Korean peninsula in September 2016. This incident confirmed that Korea is not a safe zone for earthquakes, and the industry and academia pay attention to the changes in the surface of the earth. On the other hand, overseas countries have been studying various earth surface displacements such as earthquakes, subsidence, volcanoes. And they have been conducting ground surface change researches using SAR technique of satellites. In Korea, KOMPSAT-5 was launched in August 2013 unlike Arirang1 and 2, SAR sensors were installed in the satellite. It enables to observe entire Korean Peninsula regardless of the weather. However, due to the inaccuracies of the KOMPSAT- 5 satellite orbit, there are limitations in using it to study precise ground surface displacements.

The purpose of this study is to detect displacements in terrain with different terrain ups and downs around Honam High Speed Rail. We use SAR image of German TerraSAR-X with accurate satellite orbital information. In the process, two filtering methods were tried to derive the displacement value of the surface using the optimal D-InSAR(Goldstein, Boxcar filter). The method will be settled by the type of test-bed.

The concept of SAR was first introduced by Carl wiley of Goodyear Aircraft Corporation in 1951, and in the 1980s digital image processing technology and radar interference technology were developed [1]. Now, based on data which is obtained from satellites and aircraft, it improves the precision of radar interference technology and expands its application field. Through SAR analysis, we can investigate the soil, glaciers, mining area and subsidence of grounds and landslide location [2-5].

Especially D-InSAR technique used in this study is a method of tracking displacement of the earth surface through two images. This is a powerful tool to observe microscopic surface displacements. D-InSAR was used to observe surface displacements after Etna volcanic eruption in Italy first time [6]. 
The most general study is investigating the ground statue. Some studies inquired the subsidence of ground [4]. The range of ground is various such as urban area to rural and landslide, earthquake sites [4], [7-9]. Its accuracy is determined by coherence. And, it can be applied to natural phenomenon. For instance, observing the water vapor during a heavy rain, natural vegetation, water resource management [2,3], [10-13]. In addition, there are studies which examining the InSAR process, such as coherence, phase interferogram, unwrapping, usage filter [14-18].

Recently, the further researches are conducted. This technique uses a permanent scatter which has high coherence. Radar interference technology has been developed and applied to fault zones, landslide-affected areas and urban areas. In order to observe the more quantitative and weaker surface displacements, various factors such as atmosphere and terrain effect must be considered.

However, most of the researches were performed using a single filter in the unwrap mask process, so the characteristics of each filter could not be utilized. In addition, there is a drawback which affect the low accuracy. It results from the fact that although the advantageous filters are different depending on the characteristics of terrain, it applied the same filters. In this regard, this study applied the different filters according to the terrain. Consequently, we want to select the appropriate technique to the local situation.

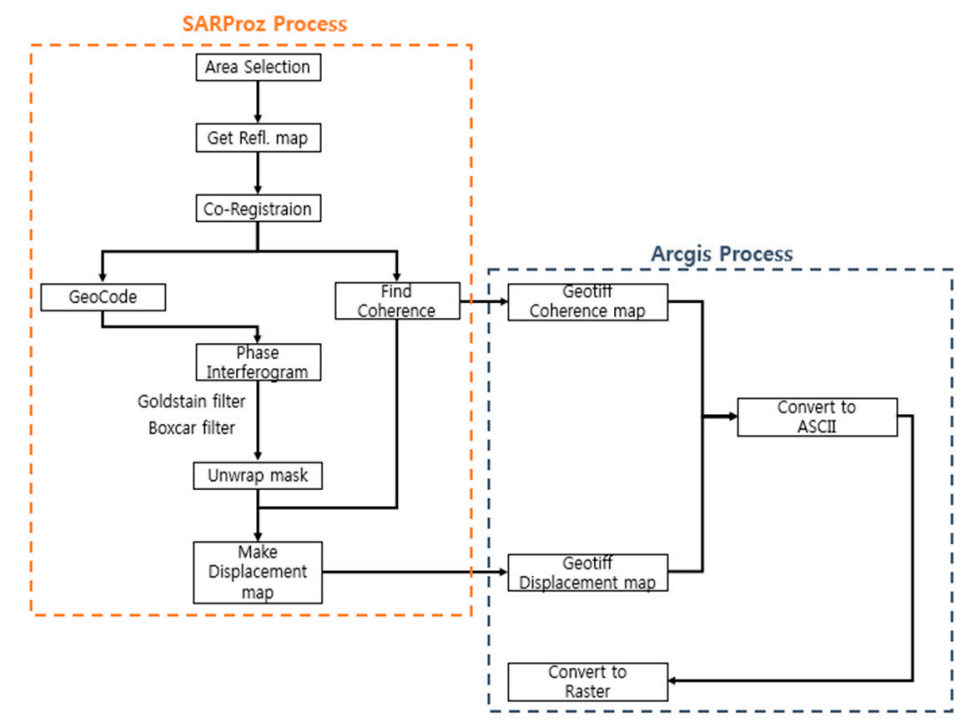

Figure 1. Research Flow chart. Left box is SARProZ Process, and right box is ArcGIS Process

\section{InSAR Process and Filtering}

\subsection{Technique of $\operatorname{In} S A R$}

Interferometric SAR technique to measure the displacement of the ground surface by making phase component with interfering two or more SAR images and unwrapping. SAR image information, transfer of satellite orbital information, reflectivity map of SAR image, matching of reflectivity map, and geocodes are called SAR process. The next step is called InSAR process, In this step, produce a differential interference by phase interferometric, and create coherence, unwrap the mask. Its purpose is deriving the selected surface displacements. Figure 2 shows these process. 
1. SAR Process

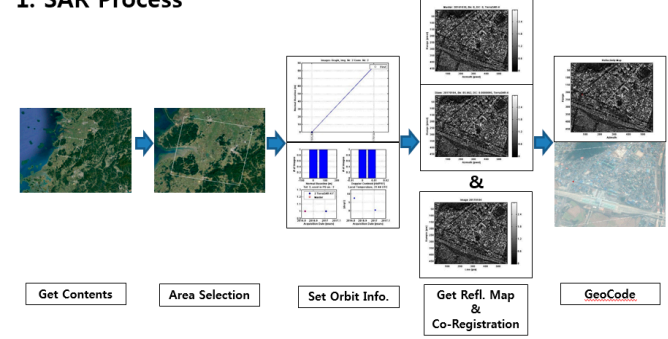

(a)
2. Interferometric SAR Process

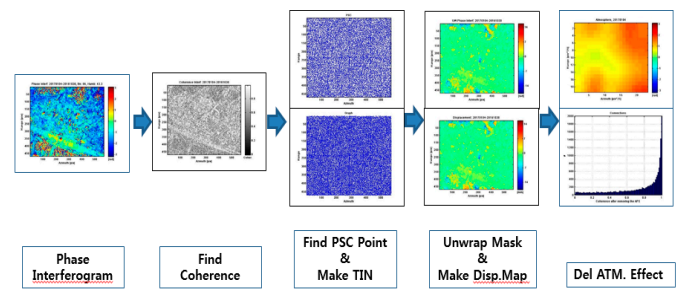

(b)

Figure 2. Research Process. (a) Main Synthetic Aperture Radar Analysis; (b) Interferometric SAR Process

\subsubsection{Reflectivity Map}

Unlike optical sensors, which use visible light, SAR image is an active sensor type using microwave to emit radio waves. In this regard the reflection of emitted radio waves is very significant in SAR image. Iron structures, asphalt and buildings have very high reflection intensity, while mountainous terrain and agricultural areas have relatively low reflection intensity. The reflection intensity also affects the band frequency of the SAR sensor. The wavelength used in SAR sensor is usually L-Band $(15-30 \mathrm{~cm})$, C-Band $(3.75 \sim 7.5 \mathrm{~cm})$ and X-Band $(2.4 \sim 3.75 \mathrm{~cm})$. There is a characteristics that the shorter the frequency, the higher the reflectance, and the longer the frequency, the higher the penetration ratio. The SAR sensor used in this study utilizes the X-band as the TerraSAR-X sensor.

As mentioned in the introduction, D-InSAR generates ground displacement by interfering two images. In this process, the main image is called master image and the secondary image is called slave image. Figure 3 shows the reflection map of the master and slave images.

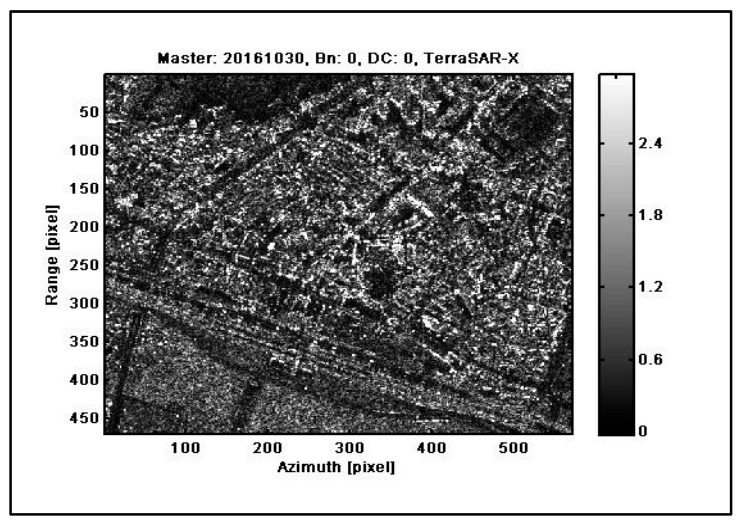

(a)

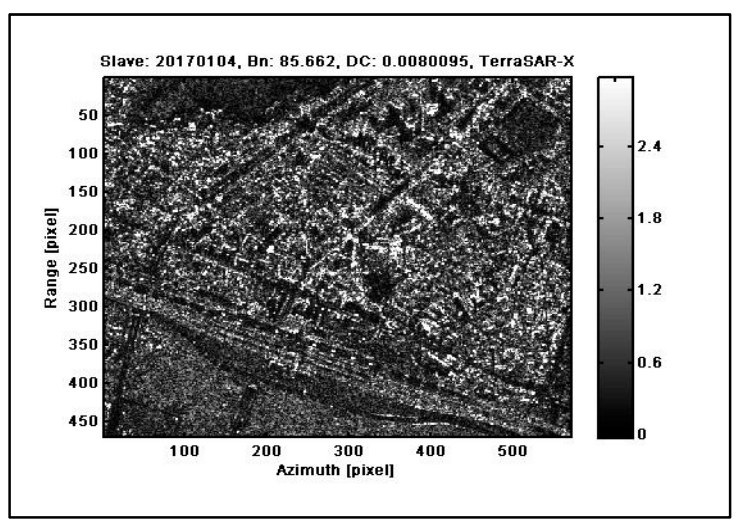

(b)

Figure 3. InSAR Reflectivity Map ; (a) Master image in Reflectivity Map Process ; (b) Slave image in Reflectivity Map Process

\subsubsection{Phase Interferogram and Coherence}

The phase component is generated by interfering with two Reflect images as shown in Fig. 5, which is called a phase interferogram. The phase is defined by the following formula:

$$
\mathrm{x}(\mathrm{t})=\mathrm{A} \cos (\omega t+\varphi)
$$


Where A indicates amplitude, $\omega$ indicates angular frequency, $\varphi$ indicates azimuth. The Phase interferogram is expressed by Radian, which is the unit of angle, and it formulates accurate surface displacement by unwrapping it [15].

As figure 4 (a) shows, it can be confirmed that the phase of the image is expressed by radian. And there are uneven patterns at the top and bottom. This is probably due to the mountainous topography of the upper part and the agricultural area below.

The InSAR technique is conducted by two radiographic images with temporal separation. The degree of changes in two images during the interval is called coherence. That is, if the coherence is high, it means that there is little change between intervals. Figure 4 (b) is an image showing coherence. The coherence has dimensionless values, and the closeness to 1 important to have high coherence. The coherence is also closely related to the accuracy of the image. Because the points with low intensities are removed by the filter in the Unwrap Mask process.

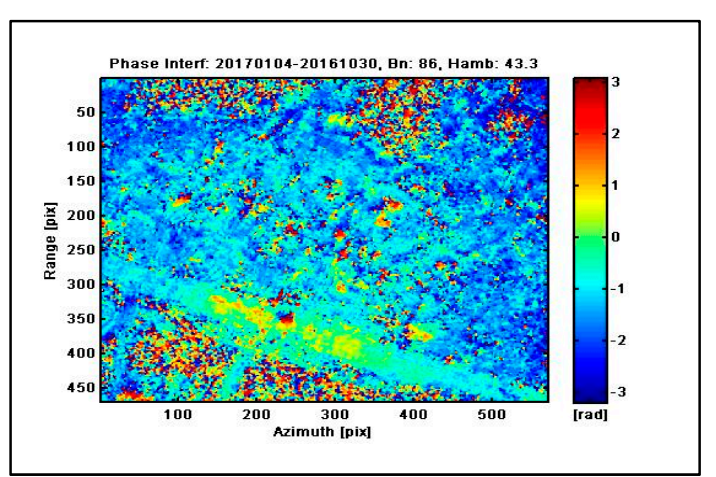

(a)

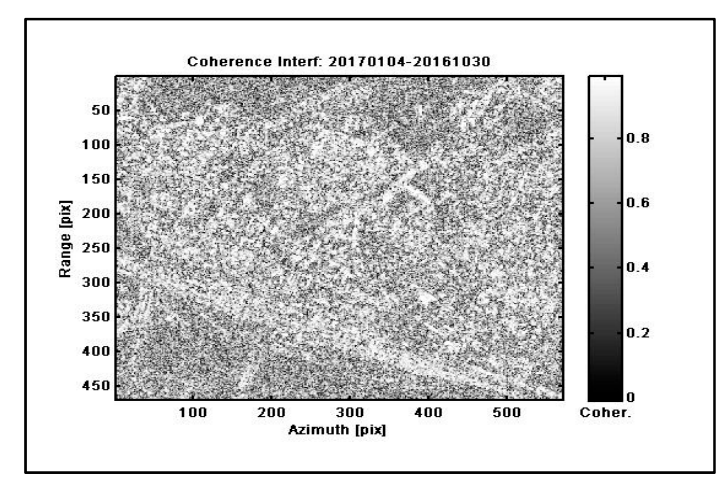

(b)

Figure 4. (a) Phase Interferogram ; (b) Coherence

\subsubsection{Unwrap mask and Displacement}

Unwrap Mask means the process of extracting the displacement by subtracting the generated interference phase [11]. The interference phase has ambiguity constrained to a value between $-\pi \sim \pi$. The measure of this is Hamb in Fig .4, which stands for Height Ambiguity [19]. It indicates ambiguity index of height value. This ambiguity is obtained by integration the phase difference between adjacent pixels. Through this process, we can obtain the relative displacement value between two images. This is called Phase Unwrap. Figure 5 (a) shows those process. As shown a figure 5 (b) image can be generated by phase unwrapping, and relative elevation value is extracted as a displacement. In other words, radian values can be converted into the unit of height through Unwrap mask. 


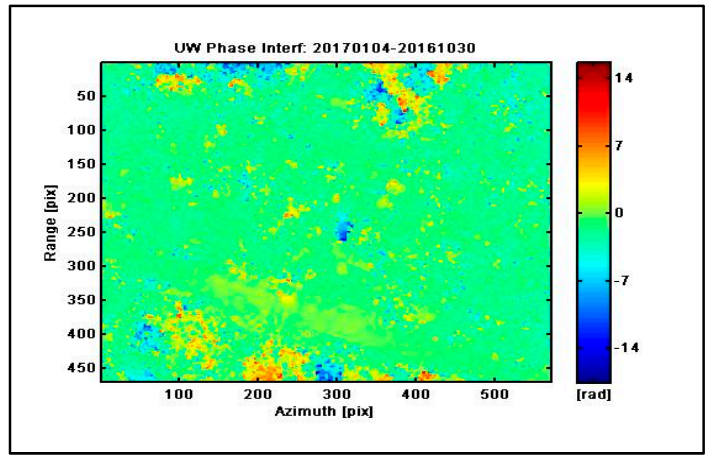

(a)

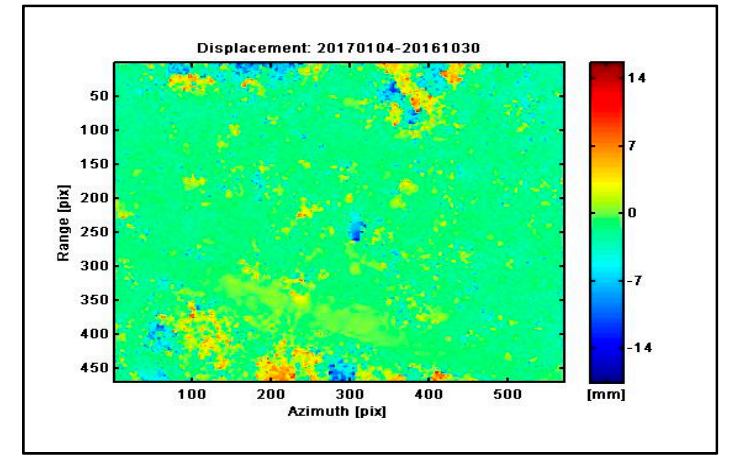

(b)

Figure 5. Unwrap mask phase interferogram and displacement. Each images have same RGB value, but different unit; (a) Unwrap mask phase interferogram (Unit : Radian); (b) displacement (Unit : Millimeter)

\subsection{InSAR Filtering Method}

\subsubsection{Goldstein}

Goldstein algorithm is a kind of the path tracking algorithm, used for unwrapping phase information and the details of the Goldstein technique are as follows $[17,18]$.

$$
\mathrm{Z}(\mathrm{u}, \mathrm{v})=\mathrm{S}\{[\mathrm{Z}(\mathrm{u}, \mathrm{v})] \alpha \cdot \mathrm{Z}(\mathrm{u}, \mathrm{v})
$$

where $\alpha$ is the filter parameter; $\mathrm{S}\{\}$ is a smoothing operator; $\mathrm{u}$ and $\mathrm{v}$ are spatial frequencies. Filtering of an interferogram is then completed by inverse Fourier transform of the smoothed Fourier samples $Z(u, v)$

The filter parameter a has a value between 0 and 1. If $\alpha=0, S\{\}$ becomes 1 and $Z(u, v)=Z(u, v)$, therefore, no filtering effect occurs. On the other hand, when $\alpha=1$, a strong filtering effect occurs, which is effective for noise reduction, however excessive smoothing occurs and adversely affects pixel accuracy. Generally, when applying the Goldstein filter on SAR images, $\alpha=0.5$ is used. In the feature of above algorithms, the amount of memory used in double operation is small and the operation time is shorter. However, in the presence of a discontinuous point (noise), there is a disadvantage in that information on the discontinuous point can not be obtained because the calculation result is dependent on the integral path. For example, if there is a two-dimensional data set as shown in Figure 6 (a), the noise components will be removed according to the path tracking method algorithm and connected linearly.

\subsubsection{Boxcar filtering}

Boxcar filter is a kind of moving average filter, which means moving average to get variation of the trend. The result of the filter matches the value of the two-dimensional multiplication in the spectral domain [18].

The characteristics of the moving average improve that the simple average can mislead the prediction of the change of the data, and the smoothing effect of the data is utilized to soften the data when the change-motion is severe. If this technique is applied, the implementation is advantageous in a flat area, but in a region with high slope, the value is unstable due to Noise-Propagation. The advantage of the Boxcar filter is that, unlike the Goldstein algorithm, noise propagation occurs, but all pixel information is available. 


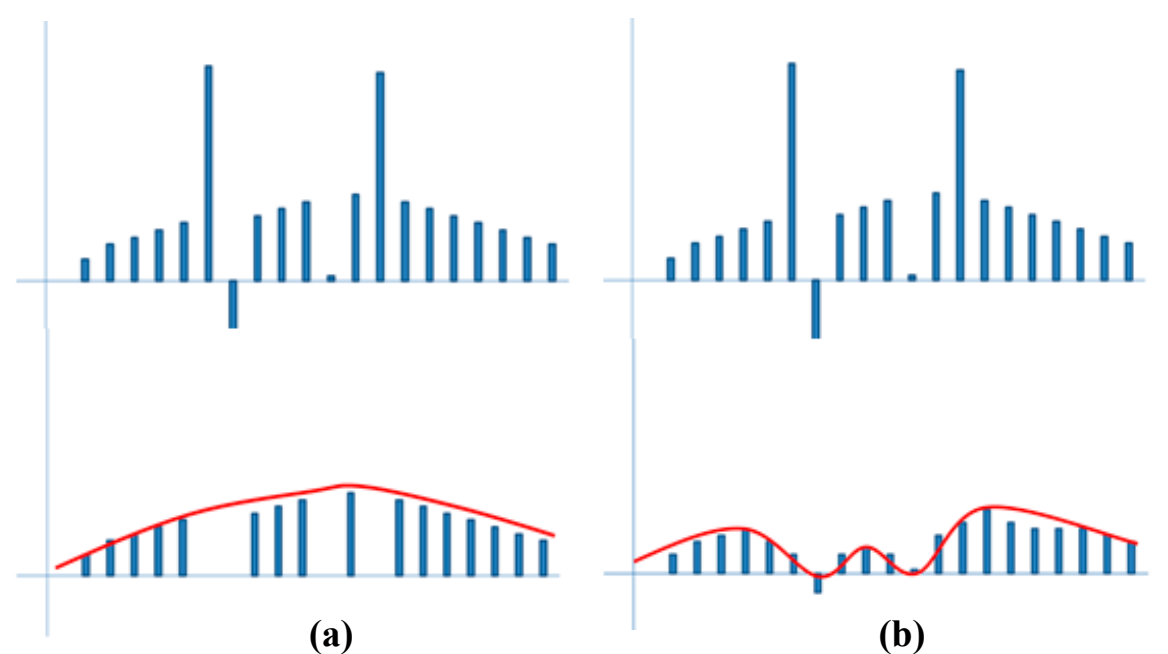

Figure 6. Two different masking filter; (a) Goldstein; (b) Boxcar

\section{Research Area and Data}

In this study, the area surrounding Jeong-eup station in Jeong-eup city, which is set as Test-Bed, is characterized by a mixture of agricultural area and urban area. In addition, the Test-bed itself is a flimsy ground with a lot of silt and clay. Due to the nature of the Honam-Plain, drought and the use of groundwater in farmland around the site, the water level is lowered and there is a high possibility of subsidence. On the other hand, the area above is the area passing through the high-speed railway (Honam line KTX), and it is an area where the factor of subsidence is large due to the micro vibration generated when the high-speed railway operates. In this study, TerraSAR-X images were used. Terra$X$ is a SAR image with a high spatial resolution of $3 \mathrm{~m}$ [20]. It has higher density of permanent scatter acquisition, sensitivity to displacement, and surface recognition rate of an object having a high roughness likewise roads, buildings than existing SAR satellite such as JERS-1, ERS-1/2 and ENVISAT. In addition, the satellite cycle (22 days) is relatively short compared to other satellites, so it has features such as quick time series data construction and mitigation of phase unwrapping problem.

In this study, two areas of testing were selected in order to compare the two filtering techniques and to improve efficiency. The first test-bed is the agricultural area and the second test-bed is the urban area. Both images were processed with the same threshold, and the Goldstein technique and the Boxcar technique were applied differently in the phase unwrap process. The first test-bed area, which is mainly agricultural area, was analyzed. The content is shown in Figure 7(a) and urban area also shown in Figure 7 (b)
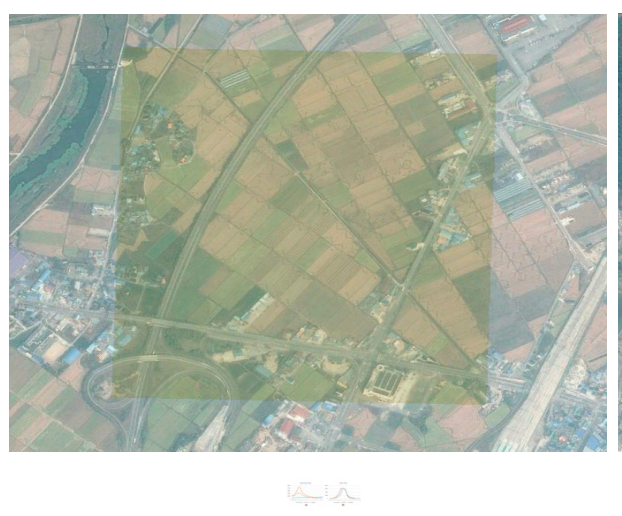

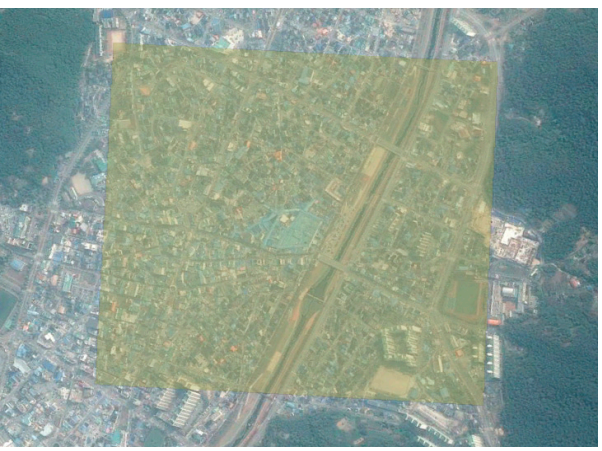

(b)

Figure 7. Test bed area: Jeong-eup station in Jeong-eup city, korea ; (a) : agricultural area; (b) : urban area 


\section{Result of study}

\subsection{Analysis of agriculture area and urban area}

As shown in Figure 8 (a), (b), (c), (d), it can be noted that the Goldstein technique is not uniform in distribution and has more noise than Boxcar technique. The reason for this is that the pixel coherence in the agricultural region are low, the pixel information cannot be acquired completely in the filtering process. On the other hand, Boxcar cannot eliminate most of the noise collectively in comparison with Goldstein technique, but because it has the advantage of utilizing most pixel information due to the influence of Value Propagation, It is confirmed that there is less displacement change.

Also as shown in Figure 8 (e), (f), (g), (h), Coherence is superior to that of agricultural area. Also, it can be confirmed that the disadvantage of boxcar filter that noise propagation does not occur in urban areas with little outliers. However, unlike the Boxcar filter, which uses the moving average filter to compute the original pixel information with adjacent pixel information, the Goldstein filter has the advantage of high accuracy because it can utilize the original pixel information as it is.

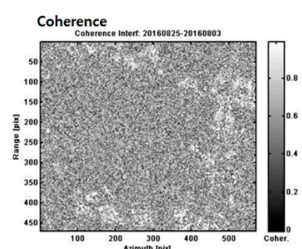

(a)

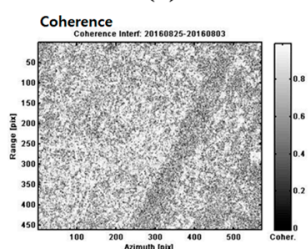

(e)

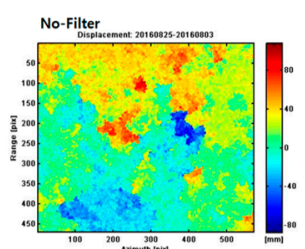

(b)

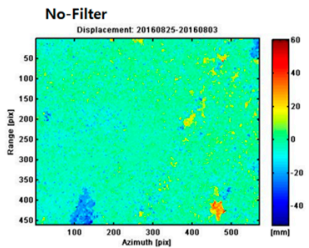

(f)

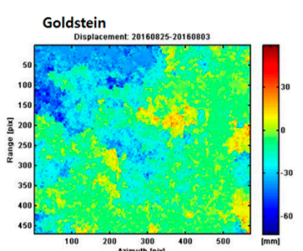

(c)

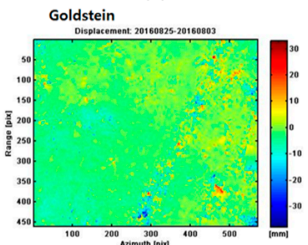

(g)

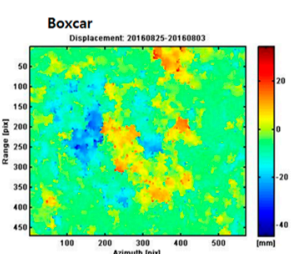

(d)

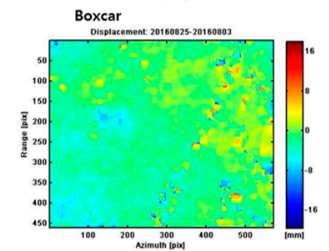

(h)

Figure 8. Coherence and displacement of each filters; (a) coherence of agricultural area; (b) agricultural area result of displacement (no-filter); (c) agricultural area result of displacement (goldstein); (d) agricultural area result of displacement (box-car); (e) coherence of urban area; (f) urban area result of displacement (no-filter); (g) urban area result of displacement (goldstein); (h) urban area result of displacement (box-car)

\subsection{Analysis of Coherence and Variance in number of points}

As shown in Table 1, it can be seen that the standard deviation of Goldstein is 2 times larger than that of Boxcar in the agricultural area. Also, the number of outliers showing about twice difference. And the number of measured point is differing 1.6 times that of boxcar compared to Goldstein. This means that the Boxcar method is more useful than the Goldstein method in agricultural area. On the other hand, the standard deviation of the urban area is similar to the Goldstein method and the Boxcar method. As can be checked from the analysis of the number of point groups, it can be seen that the urban areas do not show a considerable variation compared to the agricultural areas. Because a large outlier is not found in the process of obtaining the displacement value because the relative coherence is high.

Figure 9 Illustrates the number of points in the area. 
Table 1. Analysis of coherence and variance in number of points

\begin{tabular}{|c|c|c|c|c|c|c|}
\hline & \multicolumn{3}{|c|}{ Agricultural Area } & \multicolumn{3}{|c|}{ Urban Area } \\
\hline Filter Type & No-filter & Goldstein & Boxcar & No-filter & Goldstein & Boxcar \\
\hline Coherence AVG. & \multicolumn{3}{|c|}{0.62072} & \multicolumn{3}{|c|}{0.72648} \\
\hline Height STD. (mm) & 24.72386 & 13.77842 & 6.62068 & 2.68574 & 2.56897 & 2.61509 \\
\hline Height AVG. (mm) & 3.8082 & -12.3198 & -4.7469 & -0.5647 & -1.3332 & -1.3537 \\
\hline No. of Out-lier & 255471 & 173287 & 63219 & 12279 & 3998 & 895 \\
\hline $\begin{array}{c}\text { No. of Measuring } \\
\text { Points }\end{array}$ & 93265 & 175450 & 285518 & 306128 & 358409 & 323510 \\
\hline
\end{tabular}

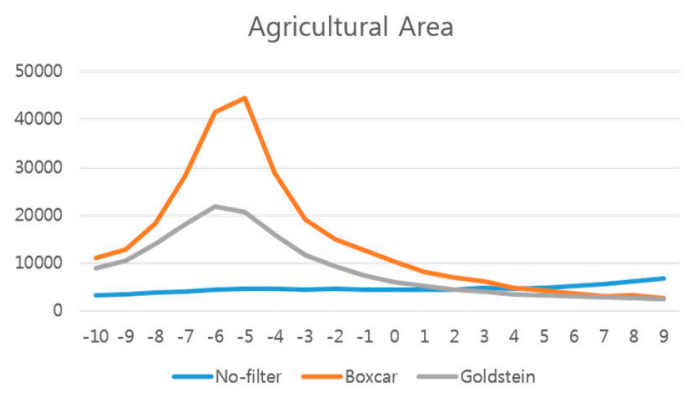

(a)

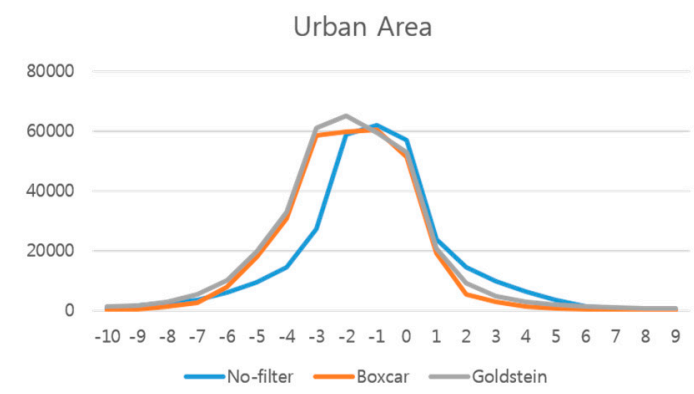

(b)

Figure 9. The number of points; (a) Agricultural Area; (b) Unban area

\section{Conclusion}

In this study, we investigated the optimal filtering technique using D-InSAR which is one of the InSAR techniques. The results are as follows.

First. The Boxcar technique is more useful than the Goldstein technique in the agricultural area. The reason for this is that in areas with low coherence, the advantage of the Boxcar technique of constructing other data through the delivery of measured values.

Second. In the urban area, the Goldstein technique is more useful than the Boxcar technique. This is due to the merit of Goldstein techniques that minimize the propagation of errors, and use pixels with strong coherence. 
Third, in order to summarize the above results, it is necessary to study the method to suppress the noise propagation caused by low coherence in the agricultural area where agricultural and urban areas are mixed.

In this study, we set up Test-Bed in urban area and agricultural area based on Coherence, applied Goldstein technique and Boxcar filter, and research to find optimal filtering algorithm. It is intended to improve the accuracy of the D-InSAR technique and is deemed useful for analyzing shortterm series analysis using less than 15 images.

\section{Refelence}

1. Carl A. Wiley. Synthetic Aperture Radars. IEEE transactions on aerospace and electronic systems 1985, 3, 440-443

2. Nolan, M.; Fatland, D.R.; Hinzman, L. DInSAR Measurement of Soil Moisture. IEEE transactions on geoscience and remote sensing 2003, 41, 2802-2813

3. Cheng, X.; Li, X.; Shao, Y.; Li, Z. DINSAR measurement of glacier motion in Antarctic Grove Mountain. Chinese science bulletin 2007, 52, 358-366

4. Hammond, W.C..; Blewitt, G.; Li, Z.; Plag, H.-P; Kreemer, C. Contemporary uplift of the Sierra Nevada, western United States, from GPS and InSAR measurements. Geology 2012, 40, 667-670

5. Li, Z. W.; Yang, Z. F.; Zhu, J. J.; Hu, J.; Wang, Y. J.; Li, P. X.; Chen, G. L. Retrieving threedimensional displacement fields of mining areas from a single InSAR pair. JOURNAL OF GEODESY 2015, 89, 17-32

6. Tizzani, P.; Castaldo, R.; Solaro, G.; Pepe, S.; Bonano, M.; Casu, F.; Manunta, M.; Manzo, M.; Pepe, A.; Samsonov, S. New insights into the 2012 Emilia (Italy) seismic sequence through advanced numerical modeling of ground deformation InSAR measurements. Geophysical research letters 2013, 40, 1971-1977

7. Wang, G.; Xie, M.; Chai, X.; Wang, L.; Dong, C. D-InSAR-based landslide location and monitoring at Wudongde hydropower reservoir in China. Environmental Earth Sciences 2013, 69, 2763 2777

8. Amarjargal, S.; Kato, T.; Furuya, M. Surface deformations from moderate-sized earthquakes in Mongolia observed by InSAR. EARTH PLANETS AND SPACE 2013, 65, 713-724

9. Liao, M.; Jiang, L.; Lin, H.;Huang, B.;Gong, J. Urban Change Detection Based on Coherence and Intensity Characteristics of SAR Imagery. Photogrammetric engineering and remote sensing 2008, 74, 9991006

10. Kinoshita, Y.; Shimada, M.; Furuya, M. InSAR observation and numerical modeling of the water vapor signal during a heavy rain: A case study of the 2008 Seino event, central Japan. Geophysical research letters $\mathbf{2 0 1 3}, 40,4740-4744$

11. Solberg, S.; Astrup, R.; Breidenbach, J.; Nilsen, B.; Weydahl, D. Monitoring spruce volume and biomass with InSAR data from TanDEM-X. Remote sensing of environment 2013, 139, 60-67 
12. Askne, J.; Santoro, M. Automatic Model-Based Estimation of Boreal Forest Stem Volume From Repeat Pass C-band InSAR Coherence. IEEE transactions on geoscience and remote sensing 2009, 47, 513516

13. Castellazzi, P.; Martel, R.; Rivera, A.; Huang, J.; Pavlic, G.; Calderhead, A. I.; Chaussard, E.; Garfias, J.; Salas, J. Groundwater depletion in Central Mexico: Use of GRACE and InSAR to support water resources management. Water resources research 2016, 52, 5985-6003

14. Andra, B.; Anura, W.; , Henschel; Michael D.; Hobbs.; Stephen.; Buehler.; Stefan A.; Ekman.; Jonas.; Lehrbass.; Brad. Seasonal variation of coherence in SAR interferograms in Kiruna, Northern Sweden. International journal of remote sensing 2016, 37, 370-387

15. Li, Z.; Zou, W.;Ding, X.;Chen, Y.; Liu, G. A Quantitative Measure for the Quality of InSAR Interferogram Based on Phase Differences. Photogrammetric engineering and remote sensing 2004, 70, 1131-1138

16. Ferretti, A.; Prati, C.; Rocca, F. Multibaseline phase unwrapping for INSAR topography estimation. Il Nuovo Cimento C 2001, 24, 2037-4909

17. Goldstein, R.M.; Werner, C.L. Radar interferogram filtering for geophysical applications. Geophysical research letters 1998, 25, 4035-4038

18. Hanssen.; Ramon. F. Radar interferometry : data interpretation and error analysis, 1st ed.; Kluwer Academic : Boston, USA, 2001

19. Krieger, G.; Moreira, A.; Fiedler, H.; Hajnsek, I.; Werner, M.; Younis, M.; Zink, M. TanDEM$\mathrm{X}$ : A Satellite Formation for High-Resolution SAR Interferometry. IEEE transactions on geoscience and

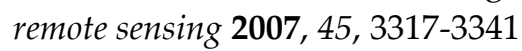

20. Stangl, M.; Werninghaus, R.; Schweizer, B.; Fischer, C.; Brandfass, M.; Mittermayer, J.; Breit, H. TerraSAR-X technologies and first results. IEE proceedings Radar, sonar, and navigation 2006, 153, 86-95

(C) 2017 by the authors. Licensee Preprints, Basel, Switzerland. This article is an open access article distributed under the terms and conditions of the Creative Commons by Attribution (CC-BY) license (http://creativecommons.org/licenses/by/4.0/). 